\title{
Changing trend of antibiotic susceptibility pattern of Enteric Fever: What is Next?
}

\author{
HAM Nazmul Ahasan, ${ }^{1}$ Homayra Tahseen Hossain, ${ }^{2}$ Ishrat Binte Reza ${ }^{3}$
}

\begin{abstract}
:
Enteric fever continues to be a major global public health problem, predominantly in the developing countries, including Bangladesh. Mortality rates due to enteric fever can be reduced from $30 \%$ to $<1 \%$ by providing effective antimicrobial therapy which is facing challenges due to emerging antimicrobial resistance. The changing trend of antibiotic susceptibility pattern of Enteric fever from pre-antibiotic era through susceptible antibiotics, then multidrug resistance (MDREF) followed by re-emergence of sensitivity to some older resistant antibiotics pose therapeutic challenges for the physicians. The purpose of this review article is to compare the antibiogram of Salmonella isolates of different recent studies and analyzing the changing trend of antibiotic resistance pattern for further successful management. This may also enable planning of antibiotic recycling wherever feasible.
\end{abstract}

Keywords: Enteric fever, antibiotic, changing trend of antibiotic, susceptibility pattern of antibiotic.

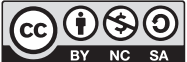

DOI: https://doi.org//0.3329/jom.v2/il.44109

Copyright: (C) 2020 Khanom M. This is an open access article published under the Creative Commons Attribution-NonCommercial-NoDerivatives 4.0 International License, which permits use, distribution and reproduction in any medium, provided the original work is properly cited, is not changed in any way and it is not used for commercial purposes.

Received: 08 August, 2019;

Accepted: 16 November, 2019

\section{Introduction:}

Nothing is constant in Medical Science- with time, the knowledge and experience is always changing. One antibiotic may be drug of choice now; but with time, it may gain resistance and become outdated. This ultimately necessitates the invention of newer drugs and newer researches. This is what actually happened in case of Enteric fever. The changing trend of antibiotic susceptibility pattern of Enteric fever from pre-antibiotic era through susceptible antibiotics, then multidrug resistance (MDREF) followed by re-emergence of sensitivity to some older resistant antibiotics pose therapeutic challenges for the physicians. The purpose of this review article is to compare the antibiogram of Salmonella isolates of different recent studies and analyzing the changing trend of antibiotic resistance pattern for further successful management. This may also enable planning of antibiotic recycling wherever feasible.

Enteric fever is an acute multi-system febrile illness transmitted by faeco-oral route, caused primarily by Salmonella enteric serotype Typhi (S. Typhi) and Paratyphi $A$ which infect only humans. Enteric fever is one of the major

1. Professor of Medicine, Popular Medical College, Dhaka

2. Associate Professor of Medicine, Popular Medical College, Dhaka

3. Assistant Professor of Medicine, Popular Medical College, Dhaka Corresponding author: Prof. HAM Nazmul Ahasan, Professor of Medicine, Popular Medical College, Dhaka.Email: ahasanhamn@, yahoo.com. public health problems in developing countries including Bangladesh, where safe drinking water and sanitation not properly ensured yet.

Worldwide, enteric fever is most prevalent in areas that are overcrowded with poor access to sanitation. Incidence estimates suggest that south-central Asia, Southeast Asia, and southern Africa are regions with high incidence of S. Typhi infection (more than 100 cases per 100,000 personyears). ${ }^{1}$ In 2000 , it was estimated that over 21.6 million (incidence of 3.6 per 1,000 population) of typhoid occurrences worldwide, resulting in 216,000 deaths and that more than $90 \%$ of this morbidity and mortality occurred in Asia. ${ }^{2}$ In an urban slum in Dhaka, the incidence of Salmonella typhi (S. typhi) was found to be $390 / 100,000$ population. ${ }^{3}$ Outbreaks are more common in summer season ${ }^{4}$ due to scarcity and contamination of drinking water. Infected and healthy carriers are the source of infection and "five Fs" (food, fingers, flies, fomites and faeces) play an important rolein the spread of the disease. ${ }^{5}$

Mortality rates due to enteric fever can be reduced from 30\% to $<1 \%$ by providing effective antimicrobial therapy which is facing challenges due to emerging antimicrobial resistance. ${ }^{6}$ Indiscriminate and injudicious use of antibiotics in undiagnosed febrile illness, sometimes by unqualified practitioners, often without laboratory support in antibiotic sensitivity test of organisms is responsible for diagnostic difficulties and antibiotic resistance. So, typhoid fever is not only a problem for patients, but also for treating physicians. 


\section{The history of antibiotic resistance:}

Antibiotic resistance is a moving target. The history of antibiotic resistance of Enteric fever can be summarized as follows:

Chloramphenicol was used universally to treat typhoid fever from 1948 until the 1970s.

Widespread resistance to Chloramphenicol occurred during 1970.

Ampicillin and trimethoprim-sulfamethoxazole (TMP-SMZ) (co-trimoxazole) then became treatments of choice.

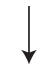

In the late 1980s, some S typhi and S paratyphi strains developed simultaneous plasmid-mediated resistance to all three of these agents (multidrug resistant [MDR] S typhi or S paratyphi).

This led to the use of fluoroquinolones in the management of enteric fever.

Afterwards, subsequent emergence of nalidixic acid resistance (NaR) and decreased susceptibility to fluoroquinolones and eventually full resistance to fluoroquinolones (Lynch 2009) occurred.

In fluoroquinolone resistant isolates, a third generation cephalosporin, frequently ceftriaxone, is often the drug of choice (Basnyat 2007) (high cost \& parenteral route of administration limit the use of Ceftriaxone).

However, sporadic cases of third generation cephalosporin resistance have been reported ${ }^{7}$. An isolate of S.typhifrom Bangladesh with high-level resistance to ceftriaxone has already been reported. ${ }^{8}$

If resistance develops against even the third generation cephalosporins, the treatment options available would be; fourth generation cephalosporins, penems, tigecycline or combination antibiotic therapy which will make treatment expensive.

However, due to restricted use of first line drugs leading to withdrawal of selective pressure, recent studies have shown that strains previously resistant to the first line drugs are showing very low or no resistance at all. ${ }^{9}$ Therefore reuse of first line drugs can be considered for management of enteric fever. ${ }^{10}$

\section{Comparison of studies:}

The antibiotic sensitivity pattern for the commonly used antibiotics was analyzed from different recent studies to detect the variations in the sensitivity pattern.

Antibiotic susceptibility pattern of Salmonella isolates:

\begin{tabular}{|c|c|c|c|c|c|c|c|}
\hline Study done by & $\begin{array}{l}\text { Reza IB } \\
\text { et al }^{11}\end{array}$ & $\begin{array}{c}\text { Sharvani R } \\
\text { et al }^{12}\end{array}$ & $\begin{array}{l}\text { Singh CL } \\
\text { et al }^{13} \\
\end{array}$ & $\begin{array}{c}\text { Chaudhary } \\
\text { et al }^{14}\end{array}$ & $\begin{array}{c}\text { Prajapati B } \\
\text { et al }^{15} \\
\end{array}$ & $\begin{array}{l}\text { Lynch } \\
\text { et } \mathrm{al}^{16}\end{array}$ & $\begin{array}{c}\text { Ahasan } \\
\text { et al }{ }^{17}\end{array}$ \\
\hline Place of study & $\begin{array}{c}\text { Dhaka, } \\
\text { Bangladesh }\end{array}$ & $\begin{array}{c}\text { India } \\
\text { (multicenter) }\end{array}$ & India & $\begin{array}{l}\text { Southern } \\
\text { India }\end{array}$ & Nepal & USA & $\begin{array}{c}\text { Khulna } \\
\text { Bangladesh }\end{array}$ \\
\hline Year of study & 2018 & 2013 & 2012-2014 & 2009-2011 & $2007-2008$ & $1999-2006$ & 1991-1992 \\
\hline Study Period & 6 months & 1 year & 27 months & 2 years & 1 year & 7 years & 1 year \\
\hline $\begin{array}{l}\text { Number of culture } \\
\text { Positive cases of Salmonella }\end{array}$ & 139 & 167 & 46 & 322 & 235 & 2016 & $48 \%$ \\
\hline \multicolumn{8}{|l|}{ ANTIBIOTIC } \\
\hline Ampicillin & $69 \%$ & $94.6 \%$ & $91.30 \%$ & $90.68 \%$ & $66.7 \%$ & $86.5 \%$ & $10.6 \%$ \\
\hline Co-trimoxazole & $75 \%$ & $95.8 \%$ & $91.30 \%$ & $95.03 \%$ & $93.5 \%$ & $86.5 \%$ & $2.1 \%$ \\
\hline Nalidixic Acid & $52 \%$ & $9.5 \%$ & $50 \%$ & $8.07 \%$ & $\ldots$ & $62.4 \%$ & \\
\hline Ciprofloxacin & $68 \%$ & $62.2 \%$ & $76.8 \%$ & $54.34 \%$ & $86.6 \%$ & $63.59 \%$ & $100 \%$ \\
\hline $3^{\text {rd }}$ generation Cephalosporin & $100 \%$ & $97 \%$ & $93.47 \%$ & $100 \%$ & $98.9 \%$ & $\ldots \ldots$ & $66.6 \%$ \\
\hline Chloramphenicol & $80 \%$ & $95.2 \%$ & $\ldots \ldots$ & $100 \%$ & $93.2 \%$ & $86.5 \%$ & $19.5 \%$ \\
\hline Imipenem & ....... & ........ & $100 \%$ & & & & \\
\hline MDREF & $30 \%$ & & & & & $13 \%$ & \\
\hline
\end{tabular}




\section{Discussion:}

Enteric fever continues to be a major global public health problem, predominantly in the developing countries, including Bangladesh. In the pre-antibiotic era, the case fatality rate due to Typhoid fever approached $20 \%$. However, after 1948, the situation changed abruptly with the discovery of powerful antibiotic Chloramphenicol. Treatment with effective antimicrobial agents - ampicillin, chloramphenicol, cotrimoxazole and later ciprofloxacin - has reduced the case fatality rate to less than $1 \% .{ }^{18}$ The ensuing 7 decades have chronicled an epic thrust-and-parry duel between the appearance of powerful new antibiotics and a wily bacterial foe's stepwise acquisition of resistance to them- now a days, this drug resistance of enteric fever is major concern rather than the disease itself.

The first multidrug-resistant strain (i.e., resistant to all the three first-line recommended drugs for treatment, i.e., chloramphenicol, ampicillin, and co-trimoxazole) emerged in Southeast Asia in the late 1980s and have since spread throughout the region. A study conducted in ICDDR,B in 2005 among 428 culture positive cases, the prevalence of MDRTF was found to be $91.4 \% .{ }^{19}$ Interestingly, several recent studies have found that strains previously resistant to the first-line drugs (chloramphenicol, ampicillin and cotrimoxazole) are now showing decreasing resistance. Thus, Variations in the sensitivity patterns reported for Salmonella isolates, stress the significance of continuous monitoring of antibiotic susceptibility pattern of locally prevalent strains.

In the present paper, we have compared 6 recent studies of different countries (including Bangladesh) regarding antibiotic susceptibility pattern of Salmonella isolates. All the studies, revealed increasing sensitivity of Salmonella isolates to the first line drugs (chloramphenicol, Cotrimoxazole \& Ampicillin) more than ciprofloxacin. The sensitivity of Chloramphenicol \& co-trimoxazole is almost similar to the sensitivity of $3^{\text {rd }}$ generation cephalosporin ( $>95 \%$ in some studies). The withdrawal of selective pressure has probably resulted in the re-emergence of sensitivity to these first-line drugs. ${ }^{20}$

In all the studies, rates of nalidixic acid resistance are of particular concern. Although this older antibacterial is rarely used for treatment, resistance to nalidixic acid can be a marker for decreased susceptibility to fluoroquinolones. Nalidixic Acid Resistance (NAR) indicates low level resistance to ciprofloxacin and results in treatment failure. In all the studies, sensitivity of Nalidixic acid and ciprofloxacin is less than the first line drugs. Hence ciprofloxacin can no longer be the keystone for treatment.
In all the studies, susceptibility to third generation cephalosporins is $>95 \%$, sometimes $100 \%$ (Reza IB et al ${ }^{11}$ Chaudhary et $\mathrm{al}^{14}$ ). A small percentage of isolates showed resistance to third generation cephalosporins $(2.9 \% \mathrm{~S}$. typhi in Sharvani $\mathrm{R}$ et $\mathrm{al}^{12}$ ), similar to study done by Jain et al., in Delhi (2\% of Salmonella enteric strains). Therefore, cephalosporins continue to be a good option for treatment of MDR and NAR cases. . The cost and parenteral route of administration, however, make ceftriaxone less suitable for patient treatment in low and middle-income countries, particularly as $60 \%-90 \%$ of enteric fever patients are treated as outpatients. An alternative would be the oral, thirdgeneration cephalosporin cefixime.

Study across five endemic Asian countries- China, India, Indonesia, Pakistan and Vietnam in the year 2008 showed 7$65 \%$ prevalence of MDR salmonella isolates. ${ }^{21}$ In the study of Sharvani R et al ${ }^{12}$, MDR was observed in $1.7 \%$ of $S$. typhi isolates, similar to studies reported by Indian Network for Surveillance, Gopal Muthu et al., in Madras, Shaik Mohammed et al., in Bangalore, World Health Organization, Jain et al., in Delhi. ${ }^{22}$ The low proportion of MDR may be due to restricted use of first line drugs leading to withdrawal of selective pressure, therefore reuse of first line drugs can be considered for management of enteric fever.

Conclusion:

There is shifting of antimicrobial susceptibility profile. Increasing resistance to quinolones is alarming so it is necessary to determine MIC levels for ciprofloxacin to avoid treatment failures. Third generation cephalosporins still remain as a drug of choice for treatment of MDR enteric fever cases. Reuse of the first line antibiotics can be considered for treating enteric fever cases. Their cheaper cost and availability in developing countries, in addition to their well-established clinical efficiency, are among the benefits of re-using chloramphenicol or ampicillin. Thus constant surveillance and vigorous audits of antibiotic sensitivity testing results are needed to determine whether the reintegration of these first-line drugs can be applied to a particular region.

\section{Conflict of interest: None.}

\section{References:}

1. Mogasale V, Maskery B, Ochiai RL, et al. Burden of typhoid fever in low-income and middle-income countries: a systematic, literature-based update with risk-factor adjustment. Lancet Glob Health 2014; 2:e570.

2. Crump JA, Luby SP, Mintz ED. The global burden of typhoid fever. Bull World Health Organ. 2004; 82(5):346353. 
3. Brooks WA, Hossein A, Goswami D, Hahar K, Alam,K, Ahmed N, et al. Bacteremic typhoid fever in children in an urban slum, Bangladesh. Emerg Infect Dis. 2005; 11(2): 326329.

4. Ratish KC, Chandrashekhar MR, Nagesha CN. An outbreak of multidrug resistant typhoid fever in Bangalore. Indian J Pediar1995; 62: 445-8.

5. Old DC, Threlfal EJ. Salmonella In: Topley and Wilson's Microbiology and Microbial Infections, 9th edition, volume 2 (Systemic Bacteriology), Arnold, London 1998, 969-97.

6. Maskey AP, Basnyat B, Thwaltes GE, et al. Emerging trends in enteric fever in Nepal: 9124 cases confirmed by blood culture 1993-2003. Trans R Soc Trop Med Hyg 2008; 102: 91-5.

7. Stoesser N, Eyre D, Basnyat B, Parry C Treatment of enteric fever (typhoid and paratyphoid fever) with third and fourth generation cephalosporins Cochrane Systematic Review - Intervention - Protocol Version published: 28 March 2013.

8. Naheed A, Ram PK, Brooks WB, Talukder KA et al. Burden of typhoid and paratyphoid fever in a densely populated urban community, Dhaka, Bangladesh. International Journal of Infectious Diseases. 2010; 14: e93.

9. Gupta V, Kaur J, Kaistha N. Re-emerging chloramphenicol sensitivity and emerging low level ciprofloxacin resistance among Salmonella enteric serotype Typhi isolates in North India. Trop Doct. 2009; 39: 28-30.

10. Mandal S, Mandal MD, Pal NK. Reduced minimum inhibitory concentration of chloramphenicol for Salmonella enteric serovartyphi. Indian J Med Sci. 2004;58:16-23.

11. Reza IB, Ahasan N HAM, Ahamed M, Hossain HT, Chowdhury T. Blood culture isolates and antibiogram of Salmonella: The gathering storm (In Press).

12. Shravani R, Hemavathi, DK. Dayanand, Poornima Shenoy , Pooja Sarmah. Antibiogram of Salmonella Isolates: Time to Consider Antibiotic Salvage.. 2016 May; 10(5): DC06DC08.
13. Singh CL, Cariappa CMP. Blood culture isolates and antibiogram of Salmonella: Experience of a tertiary care hospital 2016 Jul;72(3):281-4. doi: 10.1016/ j.mjafi.2015.07.007. Epub 2015 Sep 4.

14. Choudhary A, Gopalkrishnan R, Nambi PS, Ramasubramanian V, Ghafur KA, Thirunarayan MA. Antimicrobial susceptibility of Salmonella enterica serovars in a tertiary care hospital in southern India. Indian J Med Res. 2013; 137:800-802.

15. Prajapati B, Rai GK, Rai SK, Upreti HC, Thapa M, Singh G, Shrestha RM (2008) Prevalence of Salmonella Typhi and paratyphi infection in children: a hospital based study. Nepal Med Coll J 10: 238-241.

16. Lynch MF, Blanto EM, Bulens S, et al. Typhoid fever in the United States, 1990-2006. JAMA. 2009; 302: 859-865.

17. Ahasan N HAM, Rahman M. "Multidrug resistant type of typhoid fever in Khulna and consequent problems of treatment. Bang Med J (Khulna Branch) 1992;25:10-14.

18. Mermin JH, Townes JM, Gerber M, Dolan N, Mintz ED, Tauxe RV. Typhoid fever in the United States, 1985-1994: Changing risks of international travel and increasing antimicrobial resistance. Arch Intern Med 1998; 158 : 633-8.

19. Ahmed D, D'Costa LT, Alam K, Hussain MA Multidrug Resistant Salmonella EntericaSerovarTyphi Isolates with High-Level Resitance to Ciprofloxacin in Dhaka, Bangladesh. Antimicrobial Agents and Chemotherapy, Oct.2006, Vol 50, N0.10, p 3516-3517.

20. Gupta V, Kaur J, Kaistha N (2009) Re-emerging chloramphenicol sensitivity and emerging low level ciprofloxacin resistance among Salmonella enterica serotype Typhi isolates in North India. Trop Doct 39: 28-30.

21. Zaki SA, Karade S. Multidrug-resistant typhoid fever: a review. J Infect Dev Ctries. 2011; 5(5):324-37.

22. Indian Network for Surveillance of Antimicrobial Research Group. Antibiogram of S. enterica serovar typhi and S. enteric serovar Paratyphi A: a multi-centre study from India. WHO South-East Asia J of Public Health. 2012;1(2):182-88. 